\title{
Microbe Profile: Dictyostelium discoideum: model system for development, chemotaxis and biomedical research
}

\author{
Catherine J. Pears* and Julian D. Gross
}

\begin{abstract}
The social amoeba Dictyostelium discoideum is a versatile organism that is unusual in alternating between single-celled and multi-celled forms. It possesses highly-developed systems for cell motility and chemotaxis, phagocytosis, and developmental pattern formation. As a soil amoeba growing on microorganisms, it is exposed to many potential pathogens; it thus provides fruitful ways of investigating host-pathogen interactions and is emerging as an influential model for biomedical research.
\end{abstract}

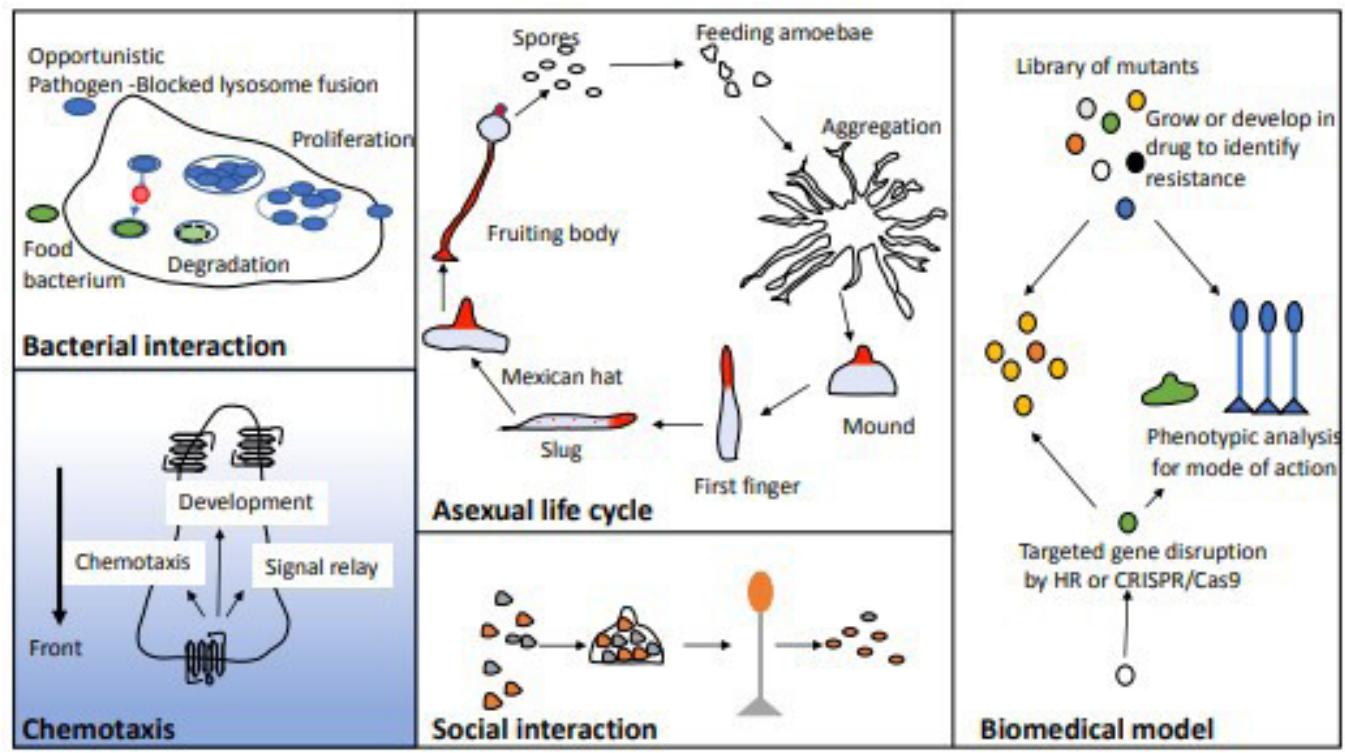

\section{Graphical abstract}

D. discoideum is an excellent system to study signalling and cytoskeletal events in chemotaxis, phagocytosis and macropinocytosis. Free-living amoebae feed on microorganisms but opportunistic pathogenic bacteria (e.g. Mycobacterium marinarum) escape degradation providing a powerful model for host-pathogen interactions. On starvation amoebae generate waves of extracellular CAMP which interacts with heterotrimeric $G$ protein-coupled receptors to generate aggregates of thousands of cells that develop into fruiting bodies containing spores held aloft by a cellular stalk. Chimaeric aggregates containing genetically distinct amoebae allow study of social conflict as some strains overpopulate the spore head, driving others into the population of dead stalk cells. Simplified versions of pathways conserved with mammals facilitate study of disease mechanisms by screening pools of mutants for drug resistance or generating targeted mutants for mode-of-action studies. 


\section{TAXONOMY}

Supergroup/phylum: amoebozoa, group: Eumycetozoa, subclass: Dictyosteliidae, order: Dictyosteliales ${ }_{2}$ family: Dictyosteliaceae, genus: Dictyostelium, species discoideum.

\section{PROPERTIES}

Dictyostelium cells are motile soil amoebae, living in forest detritus in association with many types of microorganisms, which they ingest by phagocytosis and depend on for proliferation [1]. Axenic D. discoideum derivatives with more efficient macropinocytosis are often used in the lab for ease of growth as they can be cultured in liquid broth without bacteria. The ideal growth temperature is $22^{\circ} \mathrm{C}$ with a cycle time of $8-12 \mathrm{~h}$ in axenic conditions, $4 \mathrm{~h}$ on bacteria.

Asexual development is triggered by starvation, though many extracellular factors control the transition including factors regulating growth inhibition and quorum sensing. Cells withdraw from the vegetative cell cycle and form aggregates by generating and responding chemotactically to successive waves of extracellular cAMP, and adhering via tissue-like contacts. In the subsequent mounds of up to $10^{5}$ cells, two discrete cell types emerge, the precursors of the spore and stalk cells of the fruiting bodies. A slug can form migrating towards heat and light, ensuring culmination at the surface to facilitate spore dispersal. Development is completed in around $24 \mathrm{~h}$ accompanied by death of stalk cells, sacrificing themselves for survival of spores. In some strains, food bacteria are carried into the head for dispersal with spores, an evolutionary precursor of 'farming.' The organism also has a sexual life cycle involving three mating types leading to macrocyst formation.

\section{GENOME}

D. discoideum was the first free-living protozoan to have its DNA fully sequenced. It has a genome of $34 \mathrm{Mb}$ distributed among six chromosomes with a high (77.6\%) A+T content, encoding approximately 12500 predicted proteins [2]. Ribosomal RNA genes are found on an $88 \mathrm{~kb}$ palindromic circular extrachromosomal element as well as encoded in the genome. Remarkable numbers of genes for polyketide synthases and $\mathrm{ABC}$ transporters point to the potential importance of small molecule signalling. The organism is highly resistant to DNAdamaging agents and the genome is protected by a variety of DNA repair pathways found in mammals but absent from other non-vertebrates.

As a haploid organism it is very suitable for genetic analysis and its genome is easily manipulated by homologous recombination, or CRISPR/Cas9-mediated genome editing allowing simultaneous disruption of multiple genes. There is a well-established community resource and a centralized stock/strain centre (https://.dictycr.org) including a genomewide library of insertional mutants for population screening (https://remi-seq.org).

\section{PHYLOGENY}

Amoebozoans have existed since the Neoproterozoic Era, and fossil species are found in 750 million-year-old rocks. Subclass Dictyosteliidae, order Dictyosteliales is a monophyletic assemblage within the Mycetozoa, a group including protostelid, dictyostelid, and myxogastrid slime moulds. More than 100 species of Dictyostelia have been identified, and molecular phylogeny subdivides these into four groups with representative genomes of each sequenced [3]. D. discoideum falls into the most advanced group (group 4), with additional features such as an increased number of differentiated cell types (basal disc, upper and lower cup) and using cAMP as developmental chemoattractant.

\section{KEY FEATURES AND DISCOVERIES}

D. discoideum has acquired importance as a model organism because of its genetic tractability and well-conserved cellular and developmental processes relevant to human biology and controlled by orthologous genes.

\section{A simple developmental system}

During multicellular development the stem cell-like amoebae form prestalk and prespore cells in near constant proportions. The earliest detectable expression of cell type-specific markers occurs in cells scattered within aggregative mounds, and the choice of pathway is influenced by cell cycle position and nutrient status at the time of starvation [4]. These influences lead to differential sensitivity to extracellular factors such as Differentiation Inducing Factor-1 (DIF-1), a chlorinated alkylphenone that induces differentiation of a subset of prestalk cells. Single cell RNAseq and visualisation of pulsatile transcription in single cells in vivo using GFP-tagged RNAbinding proteins provide novel ways to track these events.

\section{Social evolution}

Aggregates can form from genetically distinct amoebae, revealing 'cheaters' that are overrepresented in the final spore population and therefore the next generation [5]. This provides an ideal system for studying the evolution of conflict versus co-operation, facilitated by genome sequencing of

Received 02 October 2020; Accepted 09 February 2021; Published 01 March 2021

Author affiliations: 'Department of Biochemistry, University of Oxford, South Parks Road, Oxford OX1 3QU, UK.

*Correspondence: Catherine J. Pears, catherine.pears@bioch.ox.ac.uk

Keywords: Dictyostelium discoideum; Development; Chemotaxis; Biomedical model; Social conflict.

Abbreviations: ABC, ATP-binding cassette; cAMP, cyclic adenosine monophosphate; D. discoideum, Dictyostelium discoideum; DIF-1, differentiation

inducing factor-1; GFP, green fluorescent protein; G protein, guanine nucleotide binding protein; $h$, hours; kb, kilobases; Mb, megabases; PIP3,

phosphatidylinositol $(3,4,5)$ trisphosphate. 
increasing numbers of wild isolates. Kin discrimination, a potential defence against cheaters, is mediated by a pair of highly polymorphic adhesion proteins with differential binding activities.

\section{Motility and chemotaxis}

D. discoideum is a leading system for studying eukaryotic cell motility and chemotaxis, sometimes anticipating results in mammalian cells [6]. Multiplying amoebae locate bacterial prey by chemotaxis towards folate, and starved amoebae form aggregates by chemotaxis towards cAMP. Studies in Dictyostelium have identified key regulatory and cytoskeletal components, including the actin nucleator SCAR, and demonstrated that directional chemotaxis is achieved by biasing the location of spontaneous filopodia in a signalling network based on local excitation and long-range inhibition.

\section{Phagocytosis and host-pathogen interactions}

Dictyostelium amoebae perform efficient phagocytosis and pinocytosis, and study of these has identified cytoskeletal regulators such as PIP3 in micropinocytosis [7]. Amoebae are exposed to many possible pathogens. Opportunistic intracellular pathogens such as Legionella pneumophila and Mycobacterium marinum survive and proliferate within amoebae using molecular mechanisms also found in mammalian macrophages, and several essential components have been identified [8]. Equally important have been studies of defence mechanisms used by amoebae against bacterial infections. Zinc poisoning is a potential defence, and an orthologous iron transporter induces iron efflux from the amoebal phagosome thus starving pathogenic bacteria of iron. In the slug, sentinel cells expressing proteins related to those involved in innate immunity, such as toll-like receptors, phagocytose bacteria and accumulate toxins to protect the developing structure [9].

\section{Model for biomedical research}

The recent emphasis on replacing animals in biomedical research is increasing use of D.discoideum to study disease mechanisms, identify the mode-of-action of drugs and toxicological effects, especially during multicellular development [10]. The genome contains many genes homologous to human disease genes, allowing dissection of the biological functions of their products by targeted disruption or screening libraries of mutants for altered sensitivity to drugs during growth or development. For instance, study of orthologues of the genes implicated in neurological disorders such as Alzheimer's disease have shed light on the cellular biology underlying disease conditions. $D$. discoideum has been used to identify the targets of drugs such as lithium and valproate, and their modes of action, and novel therapeutic compounds including ones controlling epileptic seizures.

\section{OPEN QUESTIONS/MAJOR QUESTIONS}

- What is the underlying mechanism directing developmental cell fate decisions and how is proportioning and localisation established and maintained?

- How are cytoskeletal changes regulated during cell movement, phagocytosis, macropinocytosis?

- What are the mechanisms of resistance to pathogen infection and effective drug targets?

- What is the origin of self-recognition and how is it related to social conflict?

- How can D. discoideum be used most effectively to identify novel drugs and targets?

\section{Funding information}

Work in the Pears lab is funded by NC3Rs (NC/M000834/1 and Cracklt Solutions), Medical Research Council (MR/P028284/1 and ITTP studentship) and the EPA Cephalosporin Fund.

\section{Acknowledgements}

The authors would like to thank all members of the $D$. discoideum community for suggestions and support.

Conflicts of interest

The authors declare that there are no conflicts of interest.

Ethical statement

No research on animals or humans or case reports are included in this manuscript.

References

1. Kessin RH. Dictyostelium: Evolution, Cell Biology, and the Development of Multicellularity. Cambridge: Cambridge University Press; 2001.

2. Eichinger L, Pachebat JA, Glöckner G, Rajandream M-A, Sucgang R et al. The genome of the social amoeba Dictyostelium discoideum. Nature 2005:435:43-57.

3. Kawabe $Y$, Du Q, Schilde C, Schaap P. Evolution of multicellularity in Dictyostelia. Int J Dev Biol 2019;63:359-369.

4. Loomis WF. Genetic control of morphogenesis in Dictyostelium. Dev Biol 2015;402:146-161.

5. Medina JM, Shreenidhi PM, Larsen TJ, Queller DC, Strassmann JE. Cooperation and conflict in the social amoeba Dictyostelium discoideum. Int J Dev Biol 2019;63:371-382.

6. Pal DS, Li X, Banerjee T, Miao Y, Devreotes PN. The excitable signal transduction networks: movers and shapers of eukaryotic cell migration. Int J Dev Biol 2019;63:407-416.

7. Vines JH, King JS. The endocytic pathways of Dictyostelium discoideum. Int J Dev Biol 2019;63:461-471.

8. Dunn JD, Bosmani C, Barisch C, Raykov L, Lefrançois LH et al. Eat prey, live: Dictyostelium discoideum as a model for cell-autonomous defenses. Front Immunol 2017:8:8

9. Chen G, Zhuchenko 0, Kuspa A. Immune-like phagocyte activity in the social amoeba. Science 2007;317:678-681.

10. Schaf J, Damstra-Oddy J, Williams RSB. Dictyostelium discoideum as a pharmacological model system to study the mechanisms of medicinal drugs and natural products. Int J Dev Biol 2019:63:541-550. 